\title{
Prevalence of anaphylaxis and prescription rates of epinephrine auto-injectors in urban and rural areas of Korea
}

\author{
Hyunseok Cho ${ }^{1}$ and Jae-Woo Kwon ${ }^{2}$
}

\begin{abstract}
${ }^{1}$ Department of Pediatrics, Hwacheon Health Center and County Hospital, Hwacheon; ${ }^{2}$ Division of Allergy and Clinical Immunology, Department of Internal Medicine, Kangwon National University School of Medicine, Chuncheon, Korea
\end{abstract}

\author{
Received: March 3, 2018 \\ Revised : April 19, 2018 \\ Accepted: June 6, 2018

\section{Correspondence to} \\ Jae-Woo Kwon, M.D. \\ Division of Allergy and Clini- \\ cal Immunology, Department \\ of Internal Medicine, Kangwon \\ National University Hospital, \\ 156 Baengnyeong-ro, Chuncheon \\ 24289 , Korea \\ Tel: +82-33-258-9370 \\ Fax: +82-33-258-2404 \\ E-mail:legent@hanmail.net
}

\begin{abstract}
Background/Aims: Despite the clinical importance anaphylaxis and the recent increase in its occurrence, studies regarding the epidemiology of anaphylaxis, risk factors for anaphylaxis, and epinephrine auto-injector (EAI) prescription status for patients with anaphylaxis remain poorly described. Thus, we investigated the prevalence of anaphylaxis and prescription rates of EAI in urban and rural areas in Korea.
\end{abstract}

Methods: We used data from the 2010 to 2014 Health Insurance Review and Assessment database. Anaphylaxis was identified through physician-certified diagnoses using the International Classification of Diseases 1oth (ICD-10) codes ( $\mathrm{T} 780$, T782, T805, T886). Data on prescription rates of EAI were collected from the Korea Orphan \& Essential Drug Center, the only pharmacy exclusively dealing with EAI in Korea. The prescription rates of EAI were defined as the number of EAI prescribed against the number of patients with anaphylaxis.

Results: The prevalence of anaphylaxis over the 5-year period was $0.023 \%$. The annual prevalence of anaphylaxis increased over the 5 -year period. Anaphylaxis was more common in males than in females (54\% vs. $46 \%$ ) and in the population aged 50 to 59 years old. For regional analysis, urban areas showed a relatively lower prevalence of anaphylaxis (17.3 per 100,000 individuals) along with higher prescription rates (12.0\%) of EAI for patients with anaphylaxis. In contrast, rural areas showed a relatively higher prevalence of anaphylaxis (28.8 per 100,000 individuals) along with lower prescription rates (3.1\%) of EAI.

Conclusions: The prevalence of anaphylaxis has increased annually in Korea. There were regional differences in the prevalence of anaphylaxis and prescription rates of EAI between urban and rural areas in Korea.

Keywords: Anaphylaxis; Epidemiology; Clinical immunology; Prevalence; Republic of Korea

\section{INTRODUCTION}

Anaphylaxis is a serious systemic hypersensitivity reaction that can lead to sudden death [1]. Previous studies have reported that the annual prevalence of anaphylaxis was $0.01 \%$ to $0.03 \%$ and has been growing $[2,3]$. Despite the clinical importance of anaphylaxis and the recent increase in its occurrence, studies regarding the epidemiology of anaphylaxis and its risk factors remain poorly described [4]. Limited evidence suggests that the 
location of residence is associated with the incidence of anaphylaxis and epinephrine auto-injector (EAI) prescription rates [5,6]. While Sheikh and Alves [5] reported that there were more admissions for anaphylaxis in rural areas of England, Mullins et al. [6] showed that there were more EAIs sold in urban areas of Australia.

Epinephrine is the most important cure for anaphylaxis $[7,8]$. Rapid administration of epinephrine for patients with anaphylaxis is very important, as delayed administration is associated with a poor prognosis [9]. EAI is a syringe containing epinephrine and that can be immediately self-injected by patients at the occurrence of anaphylaxis, and is currently the most recommended anaphylaxis treatment for patients [10]. If there are regional variations in the prevalence of anaphylaxis and EAI prescription, different programs to prevent anaphylaxis are needed according to these different regional environments. However, there have been no epidemiologic studies evaluating the prevalence of anaphylaxis and EAI prescription rates by region.

This study was conducted in order to investigate whether there are regional differences in the occurrence of anaphylaxis and EAI prescription rates. We investigated the prevalence of anaphylaxis and EAI prescription rates among patients with anaphylaxis in urban and rural areas of Korea using the nationwide claim database.

\section{METHODS}

\section{Study population and variables}

We used the Health Insurance Review and Assessment (HIRA) database with data collected from 2010 to 2014. HIRA is a government agency that oversees all health services in Korea [11]. The National Health Insurance program in Korea began in 1977 and achieved universal application in the population by 1989. The HIRA service covers $97.0 \%$ of the Korean population (about 50 million people). The remaining $3 \%$ of the population is covered by the Medical Assistance Program. Almost all Koreans are of Korean descent. HIRA's disease statistics were collected based on hospital and pharmacy medical costs. We use the online public database provided by HIRA to show the number of patients with specific diseases each year from 2010 to 2014 by diagnosis (International Classification of Diseases [ICD] code), age group, gen- der, and regional group. Anaphylaxis diagnosis is based on a physician-certified diagnosis using ICD-10 codes consisting of anaphylactic shock due to the toxic effects of food ( $\left.\mathrm{T}_{780}\right)$, unspecified anaphylactic shock ( $\mathrm{T}_{782}$ ), anaphylactic shock by serum (T805), and anaphylactic shock due to the harmful effects of properly administered drugs or medicines (T886). The data on age reflect the patient's age at the time of the visit and were calculated using the patient's date of birth. If one patient visited the clinic both before and after their birthday, these visits were considered to be by patients of two different ages [12].

Regional classification was based on the location of the hospital where the anaphylaxis was diagnosed; the total number of administrative districts in Korea are 17, consisting of seven large cities, including one capital and six metropolitan areas, as well as 10 local provinces. The seven cities were classified as urban areas and the other regions made up of 10 local provinces were classified as rural areas [13]. The regional classification of EAI prescriptions were based on the location of the hospital that wrote the prescription. The EAI prescription data was used from the database of the Korea Orphan \& Essential Drug Center with data collected from 2010 to 2014. Since its opening in 1999, the Korea Orphan \& Essential Drug Center has been a state-owned enterprise that exclusively treats Korean EAI [14].

We examined the annual prevalence and changes in prevalence according to age, gender, and region. The prevalence of anaphylaxis was calculated by dividing the number of patients who were diagnosed with anaphylaxis by the number of residents who were reported by the National Statistical Office. The EAI prescription rates were calculated by dividing the number of EAI prescriptions by the number of patients with anaphylaxis. We studied the correlation between EAI prescription rate and the number of doctors per thousand population.

\section{Statistical analysis}

Anaphylaxis was estimated according to the patient's age, sex, and area. An independent samples $t$ test was used for comparing prevalences of anaphylaxis and a Mann-Whitney $U$ test was used for comparing EAI prescription rates. For analyzing the relationship between EAI prescription rates and the number of doctors per thousand population, Pearson correlation analysis was used. Statistical analysis was performed using SPSS for 
Windows version 19.0 (IBM Co., Armonk, NY, USA). Statistical significance was considered to exist when the $p$ value was less than 0.05 .

\section{Ethics statement}

The protocol of the present study was reviewed and approved by the Institutional Review Board of Kangwon National University School of Medicine (Reg. No. KWNUIRB-2018-02-005).

\section{RESULTS}

The prevalence of anaphylaxis over 5 years was $0.023 \%$, with the annual prevalence increasing every year (Fig. 1). Anaphylaxis prevalence was the highest at the ages of 50 to 59, and anaphylaxis was 1.2 times higher in males than in females ( $54 \%$ vs. $46 \%$ ) (Table 1). However, at ages 70 and older, anaphylaxis was more common among females.

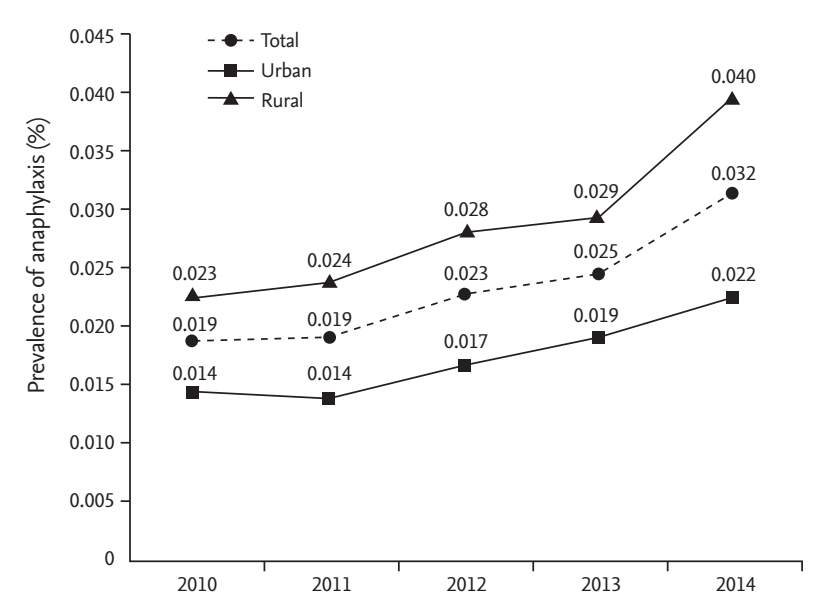

Figure 1. Annual prevalence among patients with anaphylaxis.
By region, the prevalence of anaphylaxis in rural areas was 1.7 times higher than in urban areas (17.3 vs. 28.8/100,000 person-year). The prevalence of anaphylaxis increased from $0.014 \%$ to $0.022 \%$ (57.1\% increase) in urban areas and from $0.023 \%$ to $0.040 \%$ ( $74.0 \%$ increase) in rural areas over the 5 -year period (Fig. 1). The top five areas with the highest prevalence of anaphylaxis all belonged to rural areas. Gangwon province showed the highest prevalence of anaphylaxis, which was 4.0 times higher prevalence than that of the area with the lowest prevalence (Table 2, Fig. 2). The prevalence of anaphylaxis increased over 5 years in almost all regions (Fig. 2, Supplementary Fig. 1).

The EAI prescription rate for anaphylaxis was $6.2 \%$ in Korea. The EAI prescription rate increased from 3.7\% to $7.6 \%$ over the 5 -year period, a 2.0 -fold increase. The EAI prescription rate increased from 7.8\% to $15.0 \%$ (1.9 times increase) in urban areas and from $1.4 \%$ to $3.9 \%$ (2.8 times increase) in rural areas over the 5-year period (Fig. 3). The EAI prescription rate among patients with anaphylaxis was 3.9 times higher in urban areas than in rural area (12.0\% vs. 3.1\%) (Table 2). Regional analysis between the capital and non-capital areas showed similar results when comparing between rural and urban areas. Non-capital areas had a relatively high prevalence of anaphylaxis and a low EAI prescription rate (Supplementary Table 1). The EAI prescription rate was higher in urban areas, including Seoul, which is Korea's capital city. The EAI prescription rate in the Gangwon province, which showed the highest prevalence of anaphylaxis, was onefifth of the prescription rate of Seoul (Table 2, Fig. 2). There were higher prevalences of anaphylaxis and lower EAI prescription rates in rural areas than in urban areas $(p<0.001)$. There was a moderate positive linear relationship between EAI prescription rates and the

Table 1. Age and sex distribution of patients with anaphylaxis in Korea

\begin{tabular}{|c|c|c|c|c|c|c|c|c|c|c|}
\hline & \multirow{2}{*}{ Total, \% } & \multicolumn{9}{|c|}{ Age group in years } \\
\hline & & $0-9$ & $10-19$ & $20-29$ & $30-39$ & $40-49$ & $50-59$ & $60-69$ & $70-79$ & $\geq 80$ \\
\hline \multicolumn{11}{|c|}{ Anaphylaxis $(n=59,624)$} \\
\hline Total & & 6.10 & 6.86 & 9.14 & 13.25 & 19.19 & $23 \cdot 31$ & 13.73 & 7.05 & 1.39 \\
\hline Female & 46 & 38 & 40 & 52 & 48 & 42 & 44 & 45 & 56 & 71 \\
\hline Male & 54 & 62 & 60 & 48 & 52 & 58 & 56 & 55 & 44 & 29 \\
\hline
\end{tabular}


Table 2. Annual prevalence of anaphylaxis and EAI prescription rate by region

\begin{tabular}{|c|c|c|}
\hline Region & $\begin{array}{c}\text { Prevalence of } \\
\text { anaphylaxis, } \mathrm{n}^{\mathrm{a}}\end{array}$ & $\begin{array}{c}\text { EAI prescription } \\
\text { rate, } \%\end{array}$ \\
\hline Urban area & $17 \cdot 30$ & 12.04 \\
\hline Seoul & $17 \cdot 41$ & 21.82 \\
\hline Daejeon & 25.62 & 1.59 \\
\hline Gwangju & 25.45 & 2.89 \\
\hline Ulsan & 24.44 & 5.78 \\
\hline Daegu & 14.14 & 6.21 \\
\hline Busan & 14.00 & 5.29 \\
\hline Incheon & 12.29 & 4.25 \\
\hline Rural area & 28.75 & 3.13 \\
\hline Gangwon & $49 \cdot 70$ & 4.87 \\
\hline Chungbuk & 37.04 & 3.86 \\
\hline Jeonnam & 36.33 & 0.37 \\
\hline Chungnam & 34.19 & 2.01 \\
\hline Gyeonbuk & 33.58 & 0.55 \\
\hline Jeju & 25.27 & 2.83 \\
\hline Jeonbuk & 24.99 & 1.07 \\
\hline Gyeongnam & 24.72 & 0.54 \\
\hline Gyeonggi & 23.74 & 5.38 \\
\hline Sejong & 18.14 & 0.00 \\
\hline$p$ value & $<0.001^{\mathrm{b}}$ & $<0.001^{\mathrm{c}}$ \\
\hline
\end{tabular}

EAI, epinephrine auto-injector.

${ }^{a}$ Per 100,000 person-year.

${ }^{\mathrm{b}}$ The prevalence of anaphylaxis varies between urban area and rural area.

${ }^{\mathrm{c}}$ The EAI prescription rates varies between urban area and rural area.

number of doctors per population $(r=0.623, p<0.001)$ (Supplementary Fig. 2).

\section{DISCUSSION}

The overall prevalence of anaphylaxis was $0.023 \%$ and the EAI prescription rate was $6.24 \%$ over a 5 -year period in Korea. The annual prevalence of anaphylaxis and the EAI prescription rate increased over this 5-year period. The prevalence of anaphylaxis in rural areas was
1.7 times higher than that in urban areas, but the EAI prescribing rate for patients with anaphylaxis in rural areas was as low as one-quarter of that in urban areas.

The present study showed that the annual prevalence of anaphylaxis increased over 5 years in both rural and urban areas, but the relative risk of anaphylaxis in rural areas was 1.66 times higher than that in urban areas in Korea. It is not clear whether the higher prevalence of anaphylaxis in rural areas is only found in Korea or not, but a few studies suggest that the location of residence is associated with both the incidence of anaphylaxis and EAI prescription rates [5,6]. Sheikh and Alves [5]. reported that there were regional variations in admissions for anaphylaxis in England and that the relative risk of admissions for anaphylaxis in rural areas was 1.35 times higher than that in urban areas in England. Mullins et al. [6]. suggested that residence in major cities could be a risk factor for anaphylaxis in Australia based on the higher EIA prescription rates seen in major cities than in rural areas, although they reported that there was no difference in anaphylaxis admissions between rural and urban areas. However, considering the discrepancy between EAI prescription rates and the prevalence of anaphylaxis clearly seen in the current study, the study of Mullins et al. [6] may suggest that EAI prescription rates in urban areas were much higher than those in rural areas, in spite of the similar risk of anaphylaxis admission in rural and urban areas. By using national claim data, the current study showed regional variations in the prevalence of anaphylaxis clearly comparable with the above-mentioned studies which used data on anaphylaxis admissions, because most anaphylaxis patients would be treated in emergency departments without being admitted. Considering the world-wide increase of anaphylaxis and rapid environmental changes in many countries, it is important to know that specific regional environments could generate an increased risk of anaphylaxis. Investigations on anaphylaxis epidemiology and EAI prescription by regions and planning proper programs are needed in order to prevent and manage anaphylaxis for rural and urban areas.

The reason for the higher prevalence of anaphylaxis in rural area of Korea is unclear. However, it could be postulated that the parameters involved in a regional environment, including age and gender distribution, socio-economic status, and common triggers for ana- 


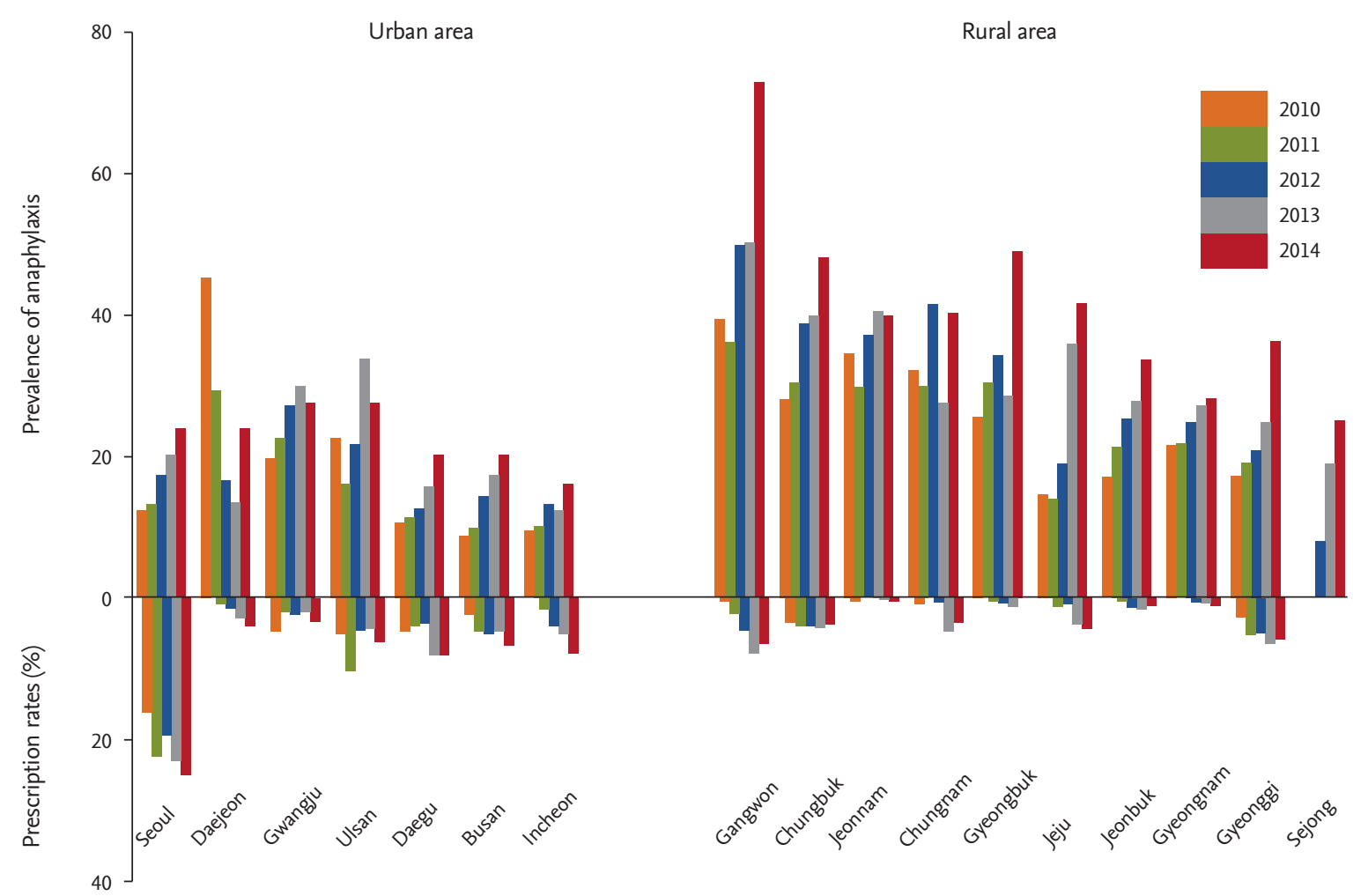

Figure 2. Annual epinephrine auto-injector prescription rates among patients with anaphylaxis.

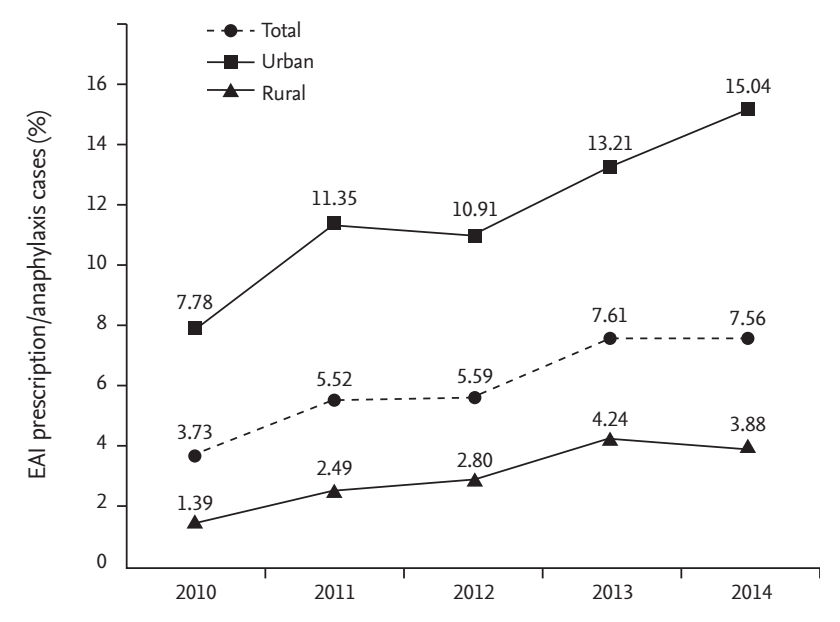

Figure 3. Annual prevalence of anaphylaxis and epinephrine auto-injector (EAI) prescription rates in urban and rural areas.

phylaxis, are closely related with regional culture and life style in rural areas, which is important. In previous studies, infants, teenagers, and the elderly have shown an increased vulnerability to anaphylaxis for different reasons [1], and the predominant triggers for anaphylaxis are different between different age groups $[1,7]$. Socio-economic status and geographic remoteness have also been suggested as risk factors for anaphylaxis admission $[5,6]$. In actuality, the common causes of anaphylaxis vary according to where the study was conducted. Studies conducted in tertiary hospitals have reported that drugs are the most common trigger, and then food and insect stings are next $[15,16]$. In contrast, studies based on general physicians' medical records reported insect stings as the most common trigger and drugs as the next-most common $[17,18]$. There may be more bees and more chances for insect stings in rural areas.

In contrast with other allergic diseases, anaphylaxis is a life-threatening condition and could happen unexpectedly, and thus some patients under attack of anaphylaxis may die due to a lack of immediate treatments [19]. EAI is critical for those patients since it is the only available form of treatment for them [10]. EAI is recommended when anaphylaxis patients who were affected an inevitable cause show respiratory symptoms or severe symptoms such as hypotension [10]. There have 
not been many studies on EAI prescription rates. Wood et al. [20] reported that $40 \%$ of patients with multiple episodes of anaphylaxis have a currently available EAI. Clark et al. [21] reported that EAI was prescribed in $27 \%$ of patients with systemic reactions to bee stings at the time of discharge from the emergency room. However, several studies have reported that EAI is under-prescribed [22]. It is reported that among patients of fatal food anaphylaxis in the United States, only $11 \%(7 / 63)$ had EAI available at the time of fatal reaction [23]. The prescription rate of EAI in Korea was lower compared with those of previous reports in this study, although it was also rising in recent years. It is partly because the diagnosis of anaphylaxis is not confirmed but based on claim data, which could include suspected and unconfirmed cases of anaphylaxis. However, the percentage of EAI prescription rates against the number of patients with anaphylaxis in urban areas was $12.04 \%$, while it was $3.13 \%$ in rural areas in Korea. It is obvious that there is a serious under-prescription of EAI in rural areas, although it is suspected that there are more triggers like bee stings which are difficult to avoid in rural areas.

The finding of the lower EAI prescription rate in rural areas compared with in urban areas in this study is consistent with that of the previous study by Mullins et al. [6] that reported a higher EAI prescription rate in urban areas and in patients with a superior social economy state in Australia. Simons et al. [24] reported that the EAI prescription rate was higher in the young population aged $<17$ years in Canada. Besides age and socio-economic status distribution in rural areas, regional variations in the medical environment including the number of allergists and accessibility to hospitals may explain the differences in EAI prescription rates. In actuality, the EAI prescription rate showed a moderate positive correlation with the number of doctors per population in the current study. The system that only allows for purchasing EAI through the Korea Orphan \& Essential Drug Center could be considered as a cause of the regional imbalance of EAI prescription rates between urban and rural areas. These findings highlight the need for education programs for the general population, patients with anaphylaxis, doctors, and emergency medical workers in rural areas.

There are several limitations to discuss. First, there were no data in this study for evaluating anaphylax- is triggers. Investigating the common triggers in rural and urban areas would be important for evaluating the differences in regional environments related to anaphylaxis. Although epidemiology studies conducted in the community have usually reported insect stings as one of the major causes of anaphylaxis, previous studies in Korea conducted in tertiary hospitals have reported drug and food as the most common causes. Second, there is no validation of the diagnosis of anaphylaxis. Insurance claim data could have many unconfirmed and anaphylaxis-like patients under the diagnosis of anaphylaxis. Also, insect sting-induced anaphylaxis could not be defined because of the absence of an appropriate ICD code. This is a common problem of studies using ICD10 codes. The data used in this paper also does not include the contents of drugs. So, by analyzing only with the specific diagnostic code, the positive predictive value may be high, but with a low specificity. In order to validate the diagnosis of anaphylaxis, reviews of the medical records and treatments are needed. Third, the area in this study is the location of the hospital where the diagnosis was made; therefore, it is not known whether the patient actually lives in an urban or rural area. However, since anaphylaxis is an urgent event, patients are likely to visit a nearby hospital. Fourth, because the data are based on insurance claim data, the diagnosis of the persons who did not go to the hospital is unknown. Although anaphylaxis is often a self-limiting disease, patients may not go to the hospital, but it is unlikely that the number of such patients would be small because of the high accessibility of medical care in Korea. Finally, it was difficult to confirm the case of recurrence using this data alone.

This study showed regional differences in the prevalence of anaphylaxis and EAI prescription rates in Korea. Rural areas showed a higher prevalence of anaphylaxis and lower EAI prescribing rates than urban areas. Differences in regional environments could not only increase the prevalence of anaphylaxis, but also create an inadequate response to anaphylaxis. The regional differences in the prevalence of anaphylaxis and EAI prescription rates need to be considered in developing anaphylaxis prevention programs and health policies of anaphylaxis. The correlation between the number of doctors per population and the EAI prescription rate showed a moderate positive relationship. This makes 
sense, as a difference in the number of doctors clearly affects the difference in EAI prescription rates. As a result, efforts will be needed to improve the number of doctors per population in order to reduce the variation in EAI prescription rates by region in the future.

\section{KEY MESSAGE}

1. Rural areas showed a higher prevalence of anaphylaxis and lower epinephrine auto-injector (EAI) prescribing rates than urban areas. Differences in regional environments could not only increase the prevalence of anaphylaxis, but also create an inadequate response to anaphylaxis.

2. The regional differences in the prevalence of anaphylaxis and EAI prescription rates need to be considered in developing anaphylaxis prevention programs and health policies of anaphylaxis.

\section{Conflict of interest}

No potential conflict of interest relevant to this article was reported.

\section{Acknowledgments}

We appreciate the Korean Health Insurance Review and Assessment service and Korea Orphan \& Essential Drug Center for providing data.

\section{REFERENCES}

1. Simons FE, Ardusso LR, Bilo MB, et al. World allergy organization guidelines for the assessment and management of anaphylaxis. World Allergy Organ J 2011;4:13-37.

2. Moneret-Vautrin DA, Morisset M, Flabbee J, Beaudouin E, Kanny G. Epidemiology of life-threatening and lethal anaphylaxis: a review. Allergy 2005;60:443-451.

3. Yang MS, Kim JY, Kim BK, et al. True rise in anaphylaxis incidence: epidemiologic study based on a National Health Insurance Database. Medicine (Baltimore) 2017;96:e5750.

4. Lieberman P, Camargo CA Jr, Bohlke K, et al. Epidemiology of anaphylaxis: findings of the American College of Allergy, Asthma and Immunology Epidemiology of Ana- phylaxis Working Group. Ann Allergy Asthma Immunol 2006;97:596-602.

5. Sheikh A, Alves B. Age, sex, geographical and socio-economic variations in admissions for anaphylaxis: analysis of four years of English hospital data. Clin Exp Allergy 2001;31:1571-1576.

6. Mullins RJ, Clark S, Camargo CA Jr. Socio-economic status, geographic remoteness and childhood food allergy and anaphylaxis in Australia. Clin Exp Allergy 2010;40:1523-1532.

7. Simons FE, Ardusso LR, Dimov V, et al. World Allergy Organization Anaphylaxis Guidelines: 2013 update of the evidence base. Int Arch Allergy Immunol 2013;162:193-204.

8. Muraro A, Roberts G, Worm M, et al. Anaphylaxis: guidelines from the European Academy of Allergy and Clinical Immunology. Allergy 2014;69:1026-1045.

9. Braganza SC, Acworth JP, Mckinnon DR, Peake JE, Brown AF. Paediatric emergency department anaphylaxis: different patterns from adults. Arch Dis Child 2006;91:159-163.

10. Ewan P, Brathwaite N, Leech S, et al. BSACI guideline: prescribing an adrenaline auto injector. Clin Exp Allergy 2016;46:1258-1280.

11. Lee SY, Jung KY, Lee IK, et al. Prevalence of treated epilepsy in Korea based on National Health Insurance data. J Korean Med Sci 2012;27:285-290.

12. Yoon HJ, Choi HY, Kim YK, Song YJ, Ki M. Prevalence of fungal infections using National Health Insurance data from 2009-2013, South Korea. Epidemiol Health 2014;36:2014017.

13. Shin J, Choi Y, Lee SG, Kim W, Park EC, Kim TH. Relationship between socioeconomic status and mortality after femur fracture in a Korean population aged 65 years and older: Nationwide retrospective cohort study. Medicine (Baltimore) 2016;95:e5311.

14. Jeong JW. Diagnosis and acute management of anaphylaxis. J Korean Med Assoc 2014;57:934-940.

15. Treudler R, Kozovska Y, Simon JC. Severe immediate type hypersensitivity reactions in 105 German adults: when to diagnose anaphylaxis. J Investig Allergol Clin Immunol 2008; 18:52-58.

16. Ye YM, Kim MK, Kang HR, et al. Predictors of the severity and serious outcomes of anaphylaxis in korean adults: a multicenter retrospective case study. Allergy Asthma Immunol Res 2015;7:22-29.

17. Peng MM, Jick H. A population-based study of the incidence, cause, and severity of anaphylaxis in the United 
Kingdom. Arch Intern Med 2004;164:317-319.

18. Helbling A, Hurni T, Mueller UR, Pichler WJ. Incidence of anaphylaxis with circulatory symptoms: a study over a 3-year period comprising 940,000 inhabitants of the Swiss Canton Bern. Clin Exp Allergy 2004;34:285-290.

19. Yang MS, Lee SH, Kim TW, et al. Epidemiologic and clinical features of anaphylaxis in Korea. Ann Allergy Asthma Immunol 2008;100:31-36.

20. Wood RA, Camargo CA Jr, Lieberman P, et al. Anaphylaxis in America: the prevalence and characteristics of anaphylaxis in the United States. J Allergy Clin Immunol 2014;133:461-467.

21. Clark S, Bock SA, Gaeta TJ, et al. Multicenter study of emergency department visits for food allergies. J Allergy
Clin Immunol 2004;113:347-352.

22. Kemp SF, Lockey RF, Simons FE; World Allergy Organization ad hoc Committee on Epinephrine in Anaphylaxis. Epinephrine: the drug of choice for anaphylaxis-a statement of the world allergy organization. World Allergy Organ J 2008;1(7 Suppl):S18-S26.

23. Bock SA, Munoz-Furlong A, Sampson HA. Fatalities due to anaphylactic reactions to foods. J Allergy Clin Immunol 2001;107:191-193.

24. Simons FE, Peterson S, Black CD. Epinephrine dispensing patterns for an out-of-hospital population: a novel approach to studying the epidemiology of anaphylaxis. J Allergy Clin Immunol 2002;110:647-651. 
Cho $\mathrm{H}$ and Kwon JW. Anaphylaxis and EAI in Korea

Supplementary Table 1. Comparison of anaphylaxis prevalence and EAI prescription rates among different regional areas in Korea over 5 years (2010 to 2014)

\begin{tabular}{lcc}
\hline & $\begin{array}{c}\text { Anaphylaxis prevalence, } \\
\text { / 100,000 person-year }\end{array}$ & EAI prescription rate, \% \\
\hline Urban area & 17.30 & 12.04 \\
Rural area & 28.75 & 3.13 \\
Capital area & 19.87 & 11.14 \\
Non-capital area & 27.09 & 2.52 \\
\hline
\end{tabular}

EAI, epinephrine auto-injector. 


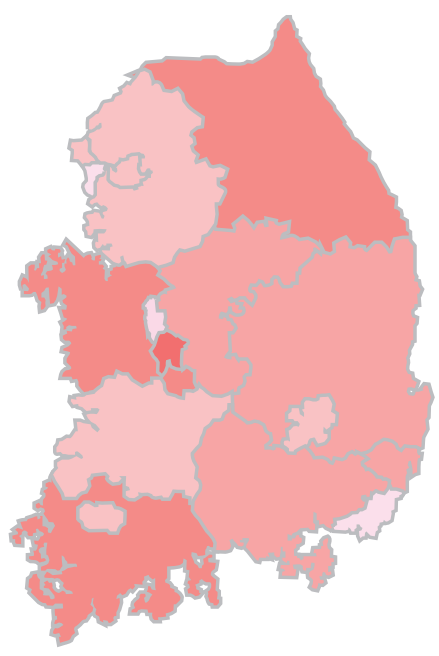

2010

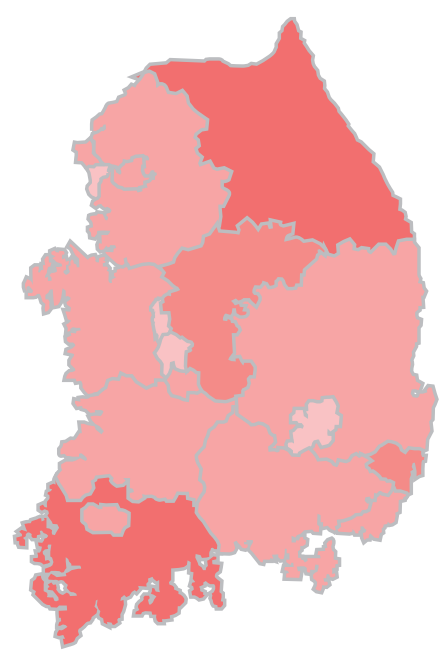

2013

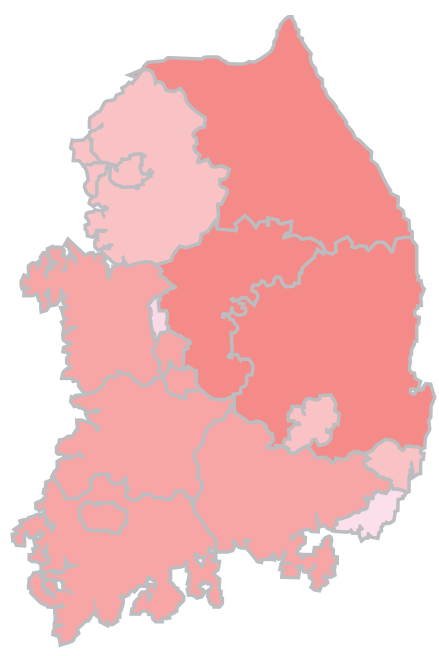

2011

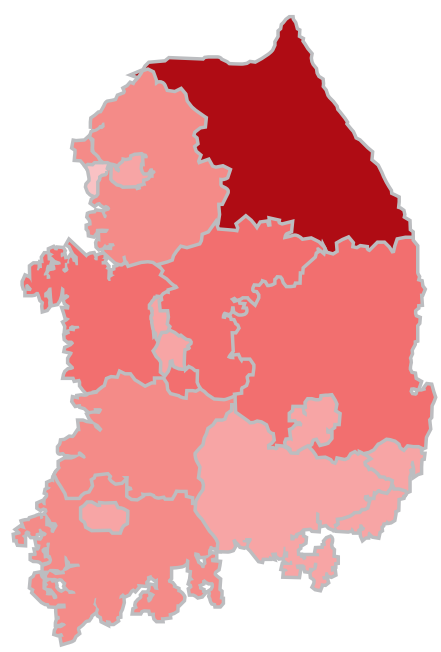

2014

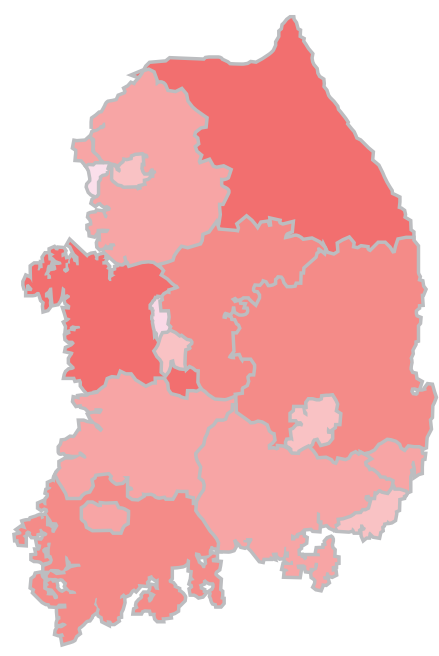

2012

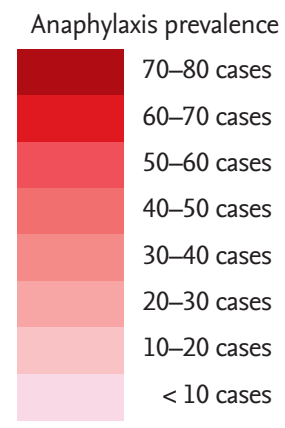

per 100,000 person-years

Supplementary Figure 1. Annual prevalence of anaphylaxis by region. The prevalence of anaphylaxis in the northeastern region (Gangwon) is increasing. 


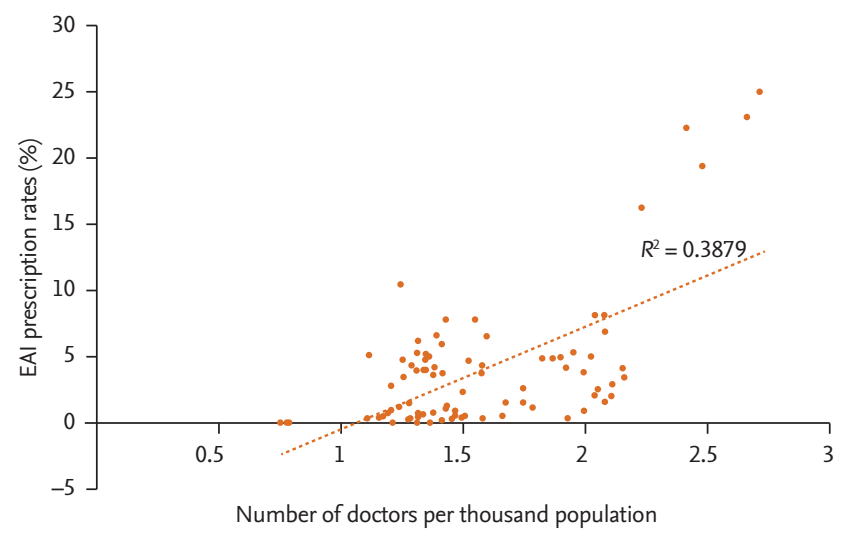

Supplementary Figure 2. Correlation between epinephrine auto-injector (EAI) prescription rates and the number of doctors per thousand population $(r=0.623, p<0.001)$. Each point represents the yearly value of specific region, and there are 17 regions and 5 years. 